He found that the associated optimum formulas had error bounds which were larger by a factor of $1 \frac{1}{2}$ or 2 than the optimum for the two parameter family we have been considering.

Our computed results indicate further that the minima in these cases are again consistent, and relatively insensitive to changes in the parameter. In practice the different differential equations had errors which corresponded to these results about as they did in the two parameter case.

Analogous results were obtained by Johnston [2] for the second and third order methods. A few special cases had been considered earlier by Kuntzmann [3].

5. Acknowledgments. We wish to thank Charles Warden who wrote some of the original programs. We are also grateful to Lockheed Aircraft and to Case Institute of Technology where the calculations were performed.

University of British Columbia, now at

University of Toronto,

Toronto 5, Ontario.

University of British Columbia, now at

Case Institute of Technology,

Cleveland, Ohio.

1. S. Gill, "A process for the step-by-step integration of differential equations in an automatic digital computing machine," Proc. Cambridge Philos. Soc., v. 47, 1951, p. 96-108.

2. R. L. Johnston, "On optimum Runge-Kutta methods for the numerical solution of ordinary differential equations," M.A. Thesis, University of British Columbia, 1961.

3. J. Kuntzmann, "Deux formules optimales du type de Runge-Kutta," Chiffres, v. 2, 1959 , p. $21-26$

4. M. Lotkin, "On the accuracy of Runge-Kutta's method," MTAC, v. 5, 1951, p. 128-132.

5. A. Ralston, "Runge-Kutta methods with minimum error bounds," Math. Comp., v. 16, 1962, p. 431-437; Corrigendum, v. 17, 1963, p. 488.

6. M. J. Romanelli, "Runge-Kutta methods for the solution of ordinary differential equations," Mathematical Methods for Digital Computers, A. Ralston and H. S. Wilf, editors, Wiley, N.'Y., 1960.

\title{
Helpful Formulas for Integrating Polynomials in Three Dimensions
}

\section{By Gilbert C. Best}

In this note formulas are given for integrating the expression $x^{a} y^{b} z^{c}$ along a line between points $P_{1}, P_{2}$, or over the surface of a plane triangle with corners at points $P_{1}, P_{2}, P_{3}$, or over the volume of a tetrahedron with corners at points $P_{1}, P_{2}, P_{3}, P_{4}$, where $P_{i}=\left(x_{i}, y_{i}, z_{i}\right)$. Since these geometric figures are of dimensions $d=1,2,3$ respectively, the writing of these formulas can be facilitated by defining "generalized volume" $V$ to be successively the length of the line, the area of the triangle or the volume of the tetrahedron in question. Then the three formulas mentioned can be given by the single equation

$$
\begin{gathered}
\int_{V} x^{a} y^{b} z^{c} d V=V \frac{a ! b ! c ! d !}{(a+b+c+d) !} \sum \prod_{i=1}^{d+1} \frac{\left(p_{i}+q_{i}+r_{i}\right) !}{p_{i} ! q_{i} ! r_{i} !} x_{i}{ }^{p_{i}} y_{i}{ }^{q_{i}} z_{i}{ }^{r_{i}} \\
d=1,2,3
\end{gathered}
$$

Received July 16, 1963. 
where the sum extends over all possible nonnegative integral values of the $p_{i}, q_{i}, r_{i}$ such that

$$
\sum_{i=1}^{d+1} p_{i}=a \quad \sum_{i=1}^{d+1} q_{i}=b \quad \sum_{i=1}^{d+1} r_{i}=c .
$$

To prove this assertion use will be made of the identity

$$
\int_{0}^{1} x^{m}(1-x)^{n} d x=\frac{m ! n !}{(m+n+1) !}
$$

which can be obtained by repeated integration by parts. Only the proof for the triangle will be given as this illustrates the general plan of the method used to prove all three cases.

In Figure 1 a triangle of base $b$ and altitude $h^{\prime}$ over points $P_{1}, P_{2}, P_{3}$ is shown. An orthogonal system of coordinates $s, s^{\prime}$ has been selected parallel and perpendicular to the base respectively, together with corresponding dimensionless variables $u$ and $v$ which vary from 0 to $1, u$ being 0 on line 1,3 and 1 on line 2,3 , while $v=0$ on line 1, 2 and 1 at point 3. This system may be oriented in any manner whatsoever with respect to the actual $X, Y, Z$ coordinates which are not shown in the figure. From an intermediate point $P_{1}{ }^{\prime}=\left(x_{1}{ }^{\prime}, y_{1}{ }^{\prime}, z_{1}{ }^{\prime}\right)$ between points $P_{1}$ and $P_{3}$ a line of length $b^{\prime}$ is drawn parallel to the base intersecting the side 2,3 at point $P_{2}^{\prime}=$ $\left(x_{2}^{\prime}, y_{2}^{\prime}, z_{2}^{\prime}\right)$.

From this it can be seen that

$$
b^{\prime}=b(1-v)
$$

hence the infinitesimal increment of area is

(4) $d A=d s d s^{\prime}=b^{\prime} d u h^{\prime} d v=b h^{\prime}(1-v) d u d v=2 A(1-v) d u d v$

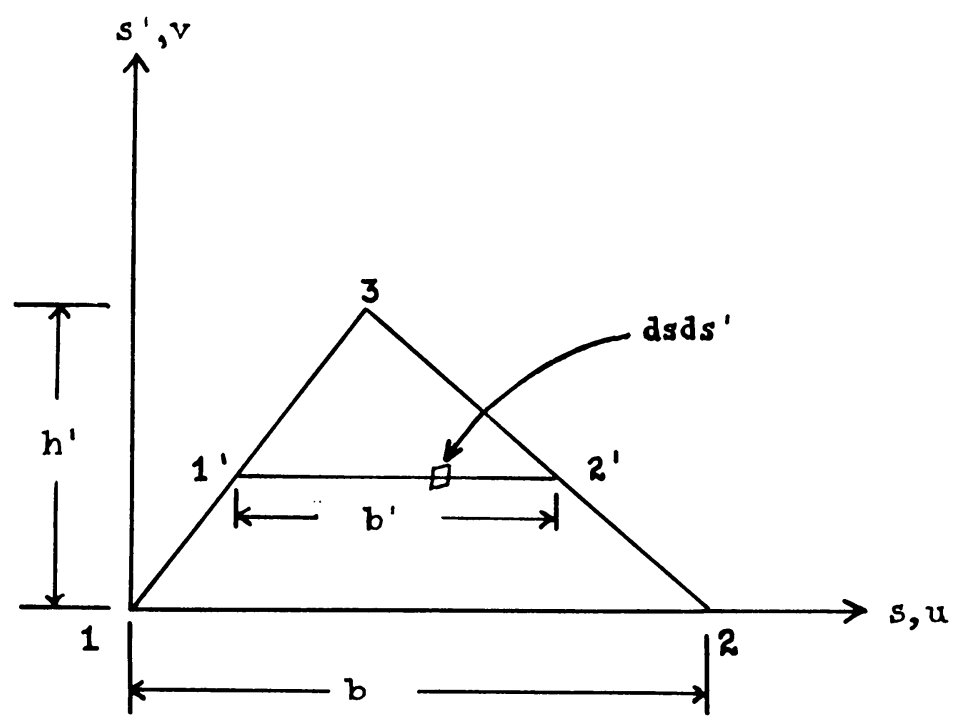

FIG. 1 
where $A=$ the area of the triangle. Also one obtains the relations

$$
\begin{aligned}
x & =x_{1}{ }^{\prime}(1-u)+x_{2}^{\prime} u \\
x_{1}^{\prime} & =x_{1}(1-v)+x_{3} v \quad x_{2}^{\prime}=x_{2}(1-v)+x_{3} v
\end{aligned}
$$

and inserting (6) into (5)

$$
x=x_{1}(1-v)(1-u)+x_{2}(1-v) u+x_{3} v
$$

with similar expressions for $y$ and $z$. Using these expressions together with the Binomial theorem and Equation (4) one obtains

$$
\begin{aligned}
\iint_{A} x^{a} y^{b} z^{c} d A & =2 A \int_{0}^{1} \int_{0}^{1} x^{a} y^{b} z^{c}(1-v) d v d u \\
& =2 A \int_{0}^{1} \int_{0}^{1}\left\{\sum \frac{a !}{p_{1} ! p_{2} ! p_{3} !} x_{1}^{p_{1}}{x_{2}}^{p_{2}} x_{3}{ }^{p_{3}}\right\}^{*}
\end{aligned}
$$

where

$$
\begin{aligned}
f(u, v)=[(1-v)(1 & -u)]^{p_{1}+q_{1}+r_{1}}[(1-v) u]^{p_{2}+q_{2}+r_{2}} v^{p_{3}+q_{3}+r_{3}}(1-v) \\
& =(1-v)^{p_{1}+q_{1}+r_{1}+p_{2}+q_{2}+r_{2}+1}(1-u)^{p_{1}+q_{1}+r_{1}} u^{p_{2}+q_{2}+r_{2}} v^{p_{3}+q_{3}+r_{3}}
\end{aligned}
$$

and

$$
\sum p_{i}=a \quad \sum q_{i}=b \quad \sum r_{i}=c .
$$

Applying Equation (2), first for $u$ and then for $v$, and using Eq. (9) gives:

$$
\int_{0}^{1} \int_{0}^{1} f(u, v) d u d v=\frac{\left(p_{1}+q_{1}+r_{1}\right) !\left(p_{2}+q_{2}+r_{2}\right) !}{\left(p_{1}+q_{1}+r_{1}+p_{2}+q_{2}+r_{2}+1\right) !} \dagger
$$

which inserted in Equation (8) and rearranging gives the desired result for the case of the triangle, i.e. $d=2$.

3703 Tulsa Way

Fort Worth, Texas

$$
\begin{aligned}
& *\left\{\sum \frac{b !}{q_{1} ! q_{2} ! q_{3} !} y_{1}^{q_{1}} y_{2}{ }^{q_{2}} y_{3}{ }^{q_{3}}\right\}\left\{\sum \frac{c !}{r_{1} ! r_{2} ! r_{3} !} z_{1}^{r_{1}} z_{2}{ }^{{ }^{2}} z_{3} z^{r_{3}}\right\} f(u, v) d v d u . \\
& \frac{\dagger\left(p_{1}+q_{1}+r_{1}+p_{2}+q_{2}+r_{2}+1\right) !\left(p_{3}+q_{3}+r_{3}\right) !}{(a+b+c+2) !}=\frac{\left(p_{1}+q_{1}+r_{1}\right) !\left(p_{2}+q_{2}+r_{2}\right) !\left(p_{3}+q_{3}+r_{3}\right) !}{(a+b+c+2) !} .
\end{aligned}
$$

